\title{
Phonon dispersion relation of metallic glasses
}

\author{
Daniel Crespo, ${ }^{1, *}$ Pere Bruna, ${ }^{1}$ Araceli Valles,${ }^{2}$ and Eloi Pineda ${ }^{1}$ \\ ${ }^{1}$ Departament de Física, Universitat Politècnica de Catalunya \\ - BarcelonaTech, 08860-Castelldefels, Catalonia, Spain \\ ${ }^{2}$ Aviation $\&$ Marine, Qatar RE, Zurich, Switzerland
}

(Dated: August 14, 2016)

\begin{abstract}
Experimental data on the phase sound speed of metallic glasses show anomalies in the Terahertz range, reflecting an underlying complex behavior of their phonon dispersion spectrum not yet explained. We determine the phonon dispersion curve of metallic glasses by means of massive molecular dynamics simulations, allowing us to obtain the low-q region behavior with unprecedented detail. Results confirm that the sound speed is constant below the THZ range, down to the macroscopic limit. On the contrary, a hardening of the sound speed, more notable in the transverse case, is found in the THZ range. This behavior is modeled in terms of a relaxation model. The model gives quantitative agreement, and allows us to determine a new threshold frequency $\omega_{h}$, at the end of the boson peak region. Above $\omega_{h}$ the shear modulus increases dramatically, reflecting the end of the amorphous-like acoustic propagation region characterized by the excess density of vibrational states.

PACS numbers: 63.50.Lm, 63.20.D-, 78.70.Ck
\end{abstract}

\section{INTRODUCTION}

The phonon spectrum reflects the mechanical properties of a material at the atomic level. In the case of metallic glasses - MGs -, from the atomic perspective ${ }^{1}$ their mechanical properties can only be described from a statistical point of view. Accordingly, the phonon spectrum of MGs gives a privileged gauge of the interplay between cohesive atomic forces and glass topology ${ }^{2}$.

The access to the phonon spectrum of metallic glasses is limited by experimental constraints. The main available experimental technique, Inelastic X-Ray Scattering (IXS) allows one to test most of the pseudo-Brillouin zone but cannot access the low wavenumber $q$ re- 
gion. Thus, there is a gap at the mesoscopic scale, between the ultrasound range accessible macroscopically and the Terahertz range accessible by IXS. In addition, experimental data of phonon dispersion and damping is restricted to the longitudinal modes, while the effects of the amorphous structure are expected to be more significant in the transverse polarizations. Furthermore, IXS measurements in MG offered contradictory results. Ichitsubo et al. ${ }^{3}$ reported a hardening on the sound speed, i.e. at low $q$ the phase velocity increases with increasing frequency, and proposed a model for fragile metallic glasses in which nanometric islands of strongly bonded regions are surrounded by a skeleton of weakly bonded regions. This model predicts a positive deviation from the linear behavior in the phonon dispersion relation at $q$ around the inverse of the size of the strongly bonded regions. That means that the sound velocity of nanometer-order wavelength should be higher than the corresponding to millimeter-order wavelength. However, later IXS experiments were not conclusive ${ }^{45}$ or even contradictory ${ }^{6}$.

Theoretical models and experimental results in other types of glasses have also predicted transitions between different regimes of phonon dispersion and damping at the nanometerscale. Schober ${ }^{7}$ stressed the importance of quasi-localized vibrations (QLV) in the phonon spectrum of glasses. The coupling of QLV would be the origin of the Boson Peak, and thus the high wavenumber regime would display hybridization between phonons and QLV. In this model the positive dispersion, i.e. the change from "soft" (lower speed) to "hard" (higher speed) phonon propagation, is observed at the frequency corresponding to the $\mathrm{BP}$ maximum. The model predicts also a change in the increase of the broadening of the phonon branch from a $\propto \omega^{4}$ to a lower power-law with exponent depending on the parameter values used in the model. A transition of the damping behavior from $\propto q^{4}$ to $\propto q^{2}$ was observed in densified $\mathrm{SiO}_{2}$, in this case attributed to a change from amorphous to crystal-like vibrational dynamics ${ }^{8}$. Positive deviation of the phonon dispersion was also observed in disordered systems like liquids where it has been explained using the mode coupling theory ${ }^{9}$.

Molecular dynamics (MD) simulations may help to fill the gap, but low wavenumbers are only accessible in very large boxes. Furthermore, most of the available results were obtained by using Lennard-Jones (LJ) or soft-sphere (SS) potentials ${ }^{101112}$. Marruzzo et al. ${ }^{12}$ simulated a binary mixture of $10^{7}$ particles with a SS potential, and showed that the spatial fluctuations of the elastic constants on a microscopic length scale are the responsible of the Boson Peak (BP) and the other elastic anomalies in the high frequency vibrational dynamics 
of glasses. In agreement with experimental ${ }^{13}$ and numerical ${ }^{10}$ results, a negative dispersion of the sound velocity and a steep increase $\left(\propto \omega^{4}\right)$ in the width of the excitation in the BP region was detected. Later, Mizuno et al. ${ }^{1415}$ used a model with SS interatomic interactions in which they can introduce several degrees of disorder and, thus, study within the same framework a fully crystalline structure and a fully amorphous one. They computed the sound velocities and, as in previous works, they found a softening of the transverse sound velocity. Furthermore, they computed the bulk and shear moduli from the sound velocity data and they found that the bulk modulus is constant and equal to the macroscopic value below the BP frequency but decreases at higher frequencies. Therefore, this result is at odds with the assumption of a frequency independent bulk modulus proposed in ${ }^{1012}$. Mizuno et al. argue that the details of the interaction potential can play a nontrivial role and cause this discrepancy. In the same line, Monaco et al. ${ }^{10}$ ask themselves which is the role that anharmonicity plays in glasses, as the LJ potentials of monoatomic glasses has been proven to be well described within the framework of the harmonic approximation ${ }^{16}$. As real glasses are anharmonic systems, the question that immediately arises is whether the LJ or the SS potentials are the proper potentials to study the dynamics of real glasses. And the contradiction remains, as until now no numerical simulation predicts the hardening of the sound speed observed experimentally.

Liquid dynamics at low particle densities (high temperatures) scales with density and temperature, showing a universal behavior weakly dependent on the particular type of interatomic potential and totally independent of the long-range attractive potential tail ${ }^{17}$. This density scaling dynamics is indeed observed between systems sharing the same type of bonding in different families of glass-formers. Contrarily, Berthier et al. ${ }^{18}$ showed that this universal density scaling is violated in the dense supercooled liquid and glassy regimes. There, the long-range details of the potential, like anharmonicity, have important effects on the dynamics of the system. The topological structure of glasses is dominated by the maximization of atomic packing and, therefore, the long-range interaction has small influence on it, but the details of the potential tail deeply affect the mechanical heterogeneity of glasses and, accordingly, their vibrational behavior ${ }^{19}$. The mesoscale mechanical heterogeneity (softstiff fluctuations) widely observed in simulations ${ }^{20}$ and already detected experimentally in metallic glasses ${ }^{21}$ is then expected to be highly dependent on the details of the particular interatomic potential of the system. 
Here we report massive molecular dynamics simulations of metallic glasses dynamics using the embedded atom method. As the details of the potential are expected to have a capital influence in the expected results, the simulations are compared to those of crystalline materials of similar compositions. Hardening of the sound speed is observed in the analyzed compositions, and a model accounting for its origin is presented.

\section{MOLECULAR DYNAMICS SIMULATIONS}

Simulations of two amorphous systems, namely $\mathrm{Zr}_{50} \mathrm{Cu}_{40} \mathrm{Al}_{10}$ and $\mathrm{Pd}_{82} \mathrm{Si}_{18}$, and a crystalline system, $\mathrm{ZrCuAl}$, were performed. The embedded atom method (EAM), originally developed by Daw and Baskes ${ }^{22}$, is a many-body potential where the total energy is given by

$$
E=\frac{1}{2} \sum_{i, j, i \neq j} \phi_{i j}\left(r_{i j}\right)+\sum_{i} F_{i}\left(\rho_{i}\right)
$$

where $\phi_{i j}$ represents the pair energy between atoms $i$ and $j$ separated by a distance $r_{i j}$, and $F_{i}$ is the embedding energy associated with an atom $i$ placed into a local site with electron density $\rho_{i}$. The electron density is calculated using

$$
\rho_{i}=\sum_{j, j \neq i} f_{j}\left(r_{i j}\right)
$$

where $f_{j}$ is the contribution to the electron charge density from atom $j$ at the location of atom i. In order to describe the interaction between the elements in the alloy the pair energy $\phi_{a b}$ between each possible pair must be supplied in addition to the electron density $\rho$ and the embedding energy $F$ for each of the elements. EAM has been successfully used in the study of metallic glasses, though it has been shown that specific potentials must be used to obtain reliable results in the amorphous state. Here we use the potentials developed for the glass state by $\mathrm{Cheng}^{23}$ and $\mathrm{Sheng}^{24}$ specifically for amorphous $\mathrm{ZrCuAl}$ and PdSi metallic glasses respectively.

Simulations were performed on cubic and high aspect ratio rectangular boxes of dimensions $l_{x}: l_{y}: l_{z}$ with $l_{x} \gg l_{y}=l_{z}$. In this way, the wavenumbers accessible on the $\mathrm{x}$ direction (long edge) are much lower than in the $\mathrm{y}$ and $\mathrm{z}$ directions (short edges), allowing us to explore the phonon dispersion and damping in the low wavenumber region without an excessive increase in the number of simulated atoms. Figure 1 shows the geometry of a 10:1:1 box. 


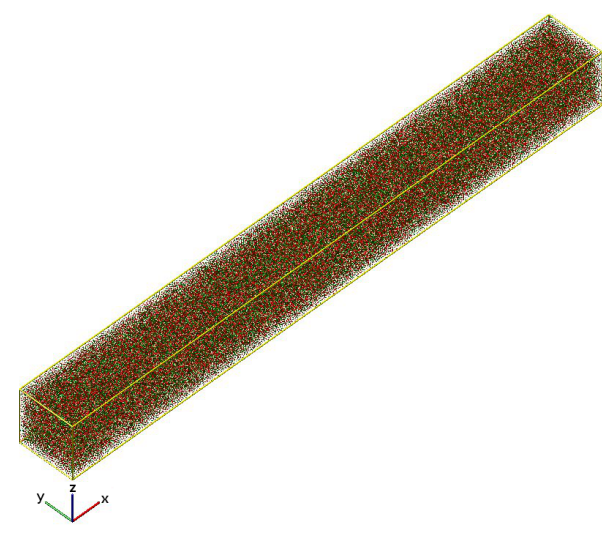

FIG. 1. Box geometry

The Large-scale Atomic/Molecular Massively Parallel Simulator (LAMMPS) code ${ }^{25}$ was used, with a timestep of 0.001 ps and periodic boundary conditions. The parameters of the simulated systems are given in Table I. In the glassy systems the liquid was equilibrated in NPT conditions at $2000 \mathrm{~K}$ for $2 \mathrm{~ns}$, then it was quenched at the desired quenching rate down to $300 \mathrm{~K}$. The glasses were subsequently equilibrated during 2 ns in NPT conditions before computing the static and dynamic structure factors, which were computed in NVT conditions for $1 \mathrm{~ns}$. The values obtained along different directions and boxes are in excellent agreement, indicating that the box shape is not influencing the phonon dynamics.

The density of states $g(\omega)$ was computed as the Fourier Transform of the velocity self correlation function, defined as

$$
C_{v}(t)=<v_{i}(0) \cdot v_{i}(t)>
$$

The static structure factor $S(q)$ was computed as the Fourier Transform of the pair distribution function $g(r)$ :

$$
g(r)=\frac{1}{N} \sum_{i \neq j}\left\langle\delta\left(r-\left|\vec{r}_{i}-\vec{r}_{j}\right|\right)\right\rangle
$$

The dynamic structure factor was computed as the self-correlation of the particle current functions $j_{\alpha}(\vec{q}, t)$,

$$
S_{\alpha}(\vec{q}, \omega)=\frac{\vec{q} \cdot \vec{q}}{2 \pi \omega^{2} N} \int d t\left\langle j_{\alpha}(\vec{q}, t) \cdot j_{\alpha}(-\vec{q}, t)\right\rangle e^{I \omega t}
$$


where $\alpha=L, T$ stands for longitudinal and transverse excitations respectively, $\vec{q}$ is the wavevector, $\omega$ is the excitation frequency, $N$ is the number of particles and

$$
\begin{aligned}
j_{L}(\vec{q}, t) & =\sum\left[\vec{v}_{i}(t) \cdot \hat{q}\right] \hat{q} e^{I \vec{q} \cdot \vec{r}_{i}(t)} \\
j_{T}(\vec{q}, t) & =\sum\left[\vec{v}_{i}(t)-\left(\vec{v}_{i}(t) \cdot \hat{q}\right) \hat{q}\right] e^{I \vec{q} \cdot \vec{r}_{i}(t)}
\end{aligned}
$$

$\vec{r}_{i}(t)$ and $\vec{v}_{i}(t)$ being the position and velocity of particle $i$ at time $t$, respectively.

In order to study the dispersion curve and the damping of the acoustic waves the Dynamic structure factor $S_{\alpha}(\vec{q}, \omega)$, computed for each wavenumber $\vec{q}$ as a function of the frequency $\omega$, was fitted to a damped harmonic oscillator response ${ }^{26}$

$$
I \propto \frac{\omega^{2} \Gamma_{q}}{\left[\Omega_{q}^{2}-\omega^{2}\right]^{2}+\omega^{2} \Gamma_{q}^{2}}
$$

where $\Omega_{q}$ is the eigenfrequency and $\Gamma_{q}$ accounts for the broadening of the phonon excitations at wavevectors of modulus $q$.

\section{RESULTS}

Figure 2 shows the static structure factor of the simulated glasses. Despite the very different compositions, $S(q)$ shows very similar features in the two glasses. The effect of the quenching rate in the amorphous structure is almost not perceived in both cases.

TABLE I. Parameters of the simulated systems.

$$
\begin{aligned}
& \text { Box size Atoms Quenching rate } \\
& \AA^{3} \quad \mathrm{~K} \cdot \mathrm{s}^{-1} \\
& \mathrm{Pd}_{82} \mathrm{Si}_{18} \text { glass } \\
& \sim 80 \times 80 \times 80, \quad 3.2 \times 10^{4} \quad 10^{13}, 10^{12} \\
& \sim 800 \times 80 \times 80,3.2 \times 10^{5} \quad 10^{13}, 10^{12} \\
& \sim 8000 \times 80 \times 80,3.2 \times 10^{6} \quad 10^{13} \\
& \mathrm{Zr}_{50} \mathrm{Cu}_{40} \mathrm{Al}_{10} \text { glass } \\
& \sim 80 \times 80 \times 80, \quad 3.2 \times 10^{4} 10^{13}, 10^{12}, 10^{10} \\
& \sim 800 \times 80 \times 80,3.2 \times 10^{5} \quad 10^{13}, 10^{12} \\
& \mathrm{ZrCuAl} \text { crystalline } \\
& \sim 800 \times 80 \times 80,3.2 \times 10^{4}
\end{aligned}
$$




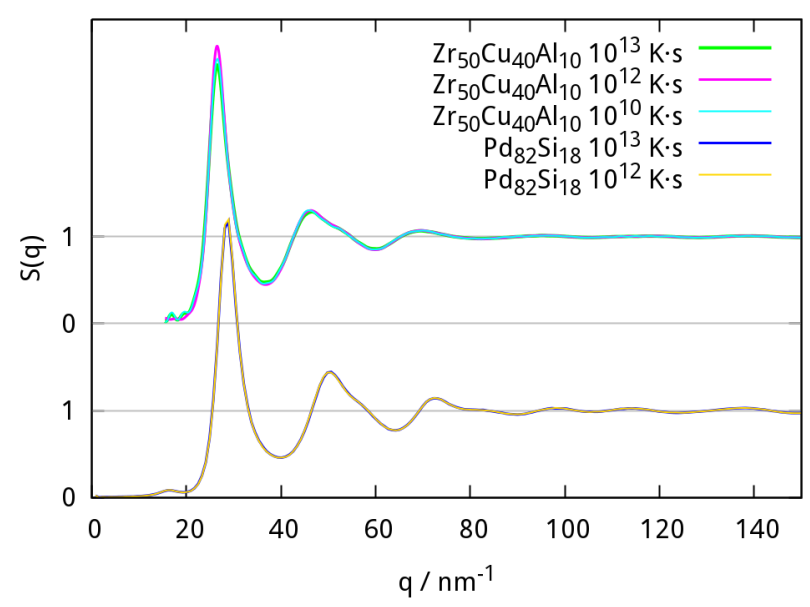

FIG. 2. Static structure factor $S(q)$ of the simulated glasses, quenched at different quenching rates.

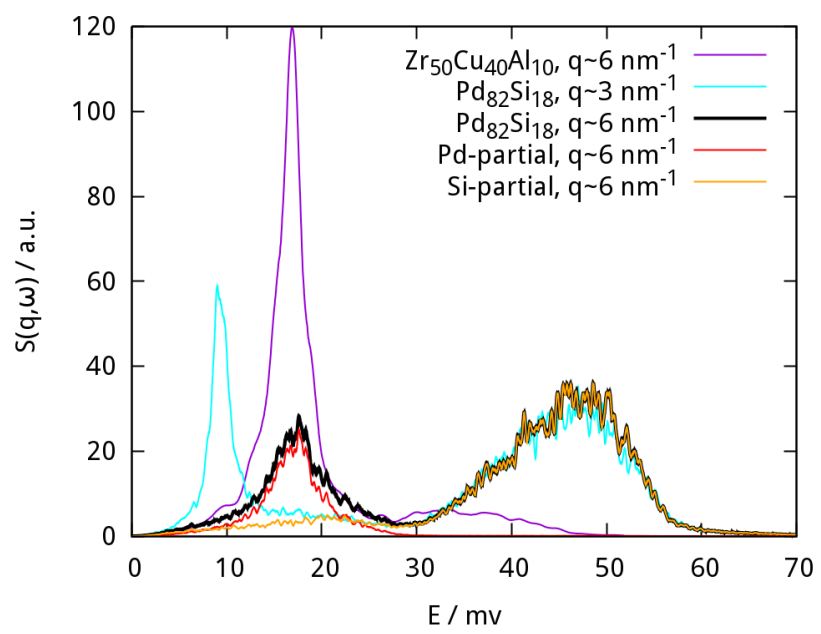

FIG. 3. Dynamic structure factor $S(\vec{q}, \omega)$ of the studied MG quenched at $10^{12} \mathrm{~K} \mathrm{~s}^{-1}$ for two particular values of $q$, and partial structure factors of $\mathrm{Pd}$ and $\mathrm{Si}$ in $\mathrm{Pd}_{82} \mathrm{Si}_{18}$ at $q \sim 6 \mathrm{~nm}^{-1}$ (see text).

Figure 3 shows a typical example of $S_{L}(\vec{q}, \omega)$ in the studied MGs. While $S_{L}(\vec{q}, \omega)$ is single peaked in $\mathrm{Zr}_{50} \mathrm{Cu}_{40} \mathrm{Al}_{10}$, it shows a secondary resonance in $\mathrm{Pd}_{82} \mathrm{Si}_{18}$ at higher frequencies. $S_{T}(\vec{q}, \omega)$ shows the same behavior. To understand the origin of this second resonance, $S_{L}(\vec{q}, \omega)$ is plotted for two values of $q$ in this case. The low-frequency resonance behaves as the expected acoustic branch; the resonance frequency increases with the wavenumber and its intensity decreases as $q$ increases. On the contrary, the high-frequency resonance has an almost constant shape, independent of $q$. Its low intensity makes it difficult to detect at low $q$ 


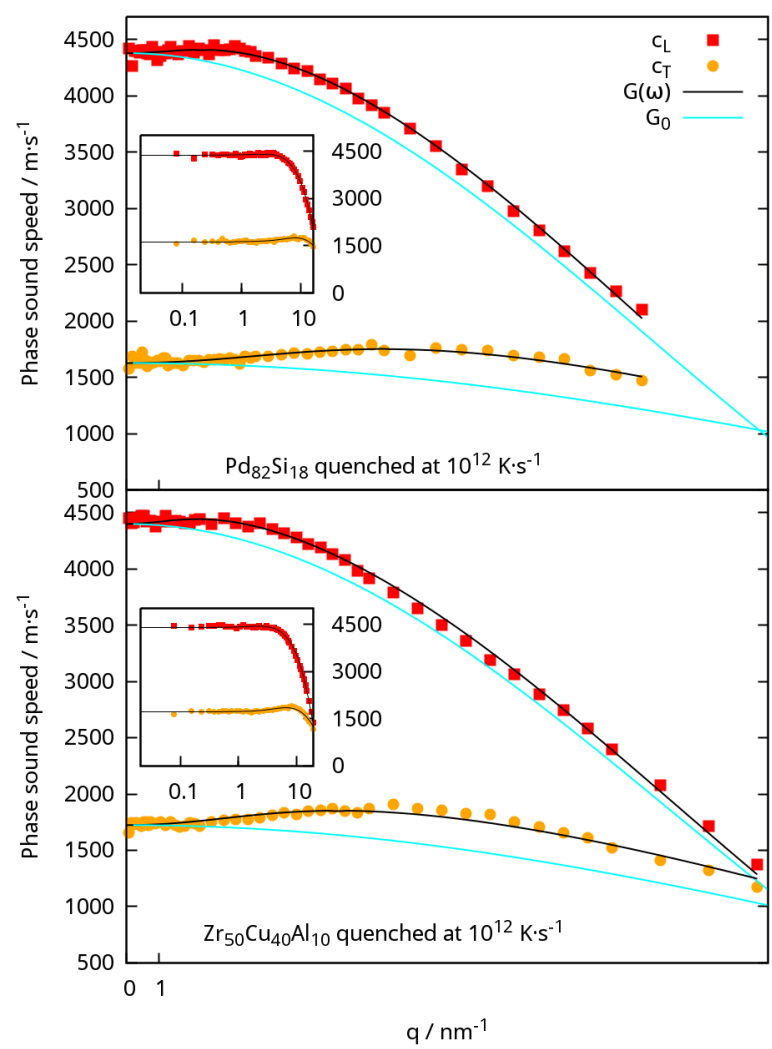

FIG. 4. (color online) Longitudinal $\left(c_{L}\right)$ and transverse $\left(c_{T}\right)$ phase sound speeds of the simulated glasses in linear and semi-logarithmic scale (inset) to show the behavior in the $q \rightarrow 0$ (macroscopic) limit. Solid lines correspond to the model developed in section IV

although it is always present. The partial dynamic structure factors, also shown in the figure, show that only $\mathrm{Si}$ atoms contribute to the high-frequency resonance. This indicates that rather than an optical phonon branch, this resonance is associated to Einstein oscillators created by a sort of caging effect of the Si atoms. Note that the corresponding Einstein temperature is about $550 \mathrm{~K}$, while the glass is being simulated at $300 \mathrm{~K}$. Einstein oscillators were already reported in $\mathrm{MGs}^{27}$, but with much lower energies. In these cases they were associated to phonon localized modes, while here they seem to correspond to individual atomic movements. However, Hosokawa reported recently unexpected vibrational modes in $\mathrm{Pd}_{40} \mathrm{Ni}_{40} \mathrm{P}_{20}$ in the same range of energies ${ }^{28}$, which may have a similar origin. This effect is most probably due to the large mass difference between $\mathrm{Pd}$ and $\mathrm{Si}$, as $\mathrm{m}_{\mathrm{Pd}} / \mathrm{m}_{\mathrm{Si}} \sim 3.8$.

Figure 4 shows the phase sound speeds $c_{L}$ (longitudinal) and $c_{T}$ (transverse) computed in $\mathrm{Pd}_{82} \mathrm{Si}_{18}$ and $\mathrm{Zr}_{50} \mathrm{Cu}_{40} \mathrm{Al}_{10}$ vitrified at a quenching rate $(\mathrm{QR})$ of $10^{12} \mathrm{~K} \mathrm{~s}^{-1}$. Similar plots 

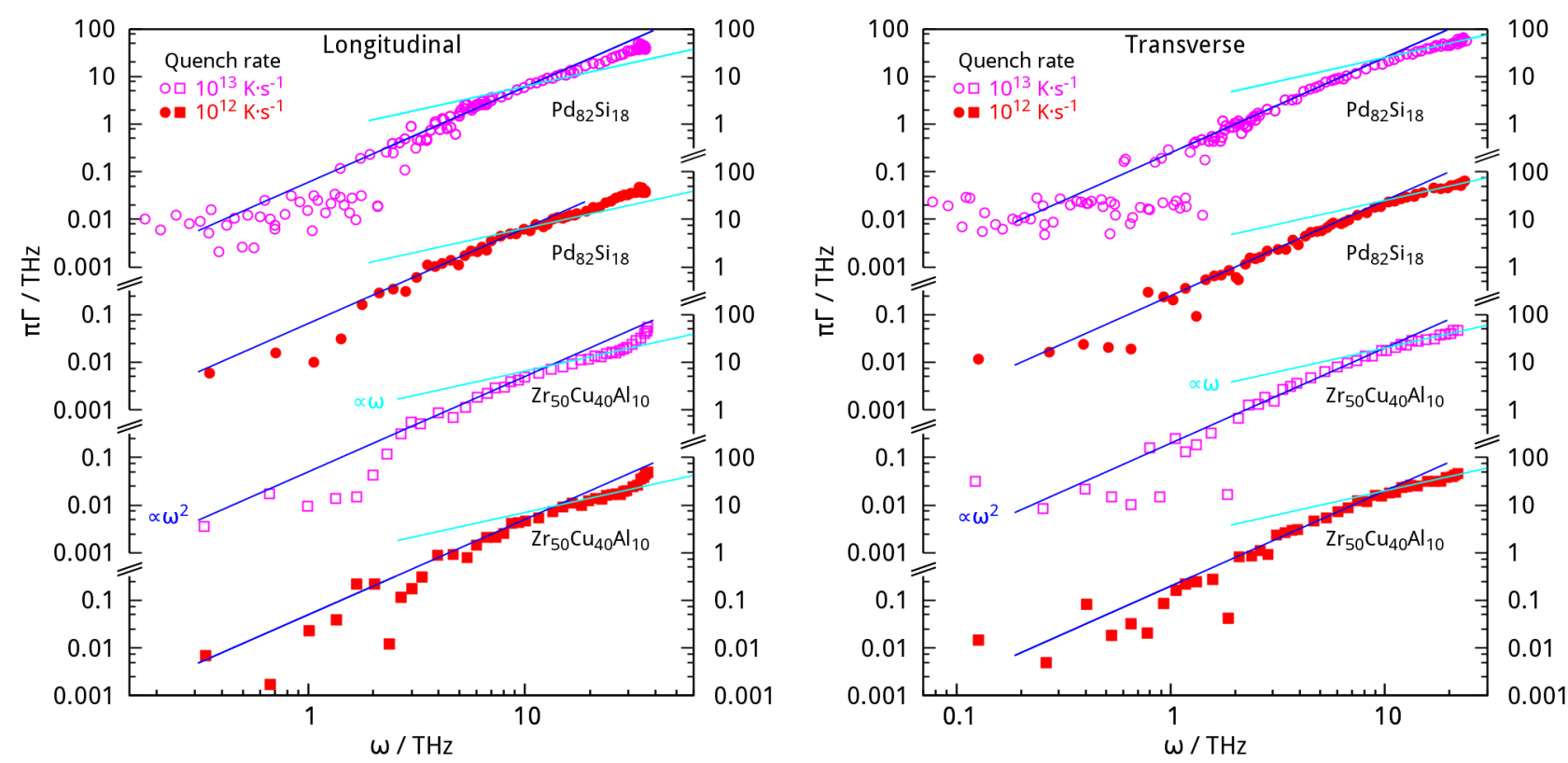

FIG. 5. Phonon disperson plots of longitudinal - left - and transverse - right - excitations.

for the remaining quenching rates are given in the supplementary materials. Both $c_{L}$ and $c_{T}$ show a constant value in the macroscopic limit, as shown in the figure inset. This is the only possible physically meaningful result; otherwise, the macroscopic sound speed should show some kind of anomaly. At increasing wavenumbers, $q>1 \mathrm{~nm}^{-1}$, a hardening on both speeds is noticed. The maximum on the hardening appears at larger wavenumbers for transverse than for longitudinal excitations. This fact contrast with pseudo-potential simulations of $\mathrm{MG}^{2930}$ and in monoatomic LJ glasses ${ }^{10}$, where a direct correlation between the longitudinal and transverse sound speed was found by assuming a constant bulk modulus.

Figure 5 shows the frequency dependence of sound attenuation coefficients for longitudinal and transverse excitations. The broadening of the transverse acoustic excitation shows a linear dependence on $\omega$ at high frequencies, while it changes to a quadratic dependence when approaching the macroscopic limit $(\omega \rightarrow 0)$, as expected for propagating waves in an amorphous homogeneous media. Similar dependencies are expected in longitudinal excitations, but they are no clear in $\mathrm{Pd}_{82} \mathrm{Si}_{18}$. This is probably due to the effect of the above cited Einstein oscillators, which affect the high frequency behavior of the acoustic excitations. It is worth to mention that due to the small size of the box the data for the quenching rate of $10^{10}$ $\mathrm{K} \cdot \mathrm{s}^{-1}$ do not allow to determine the $\omega^{2} / \omega$ crossover in this case. It has been largely discussed 


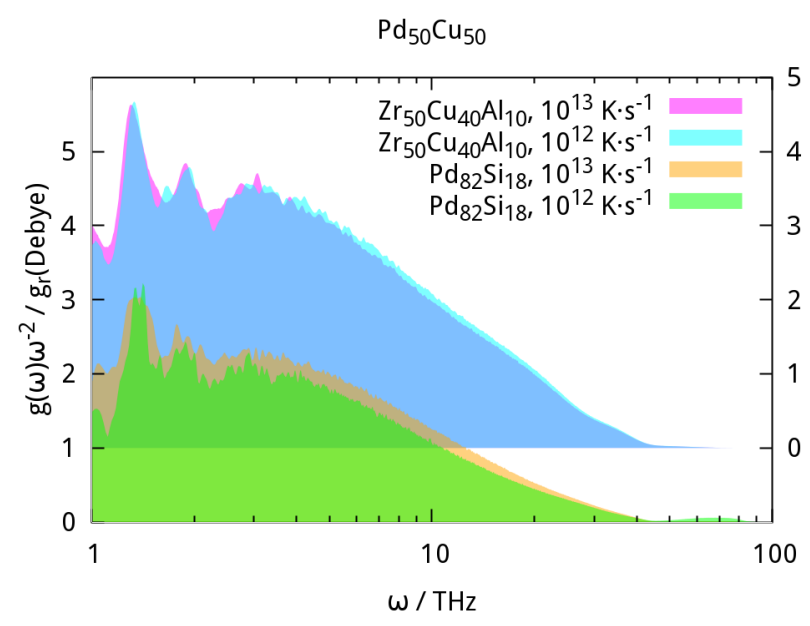

FIG. 6. Reduced density of states of the simulated glasses showing the intensity of the Boson peak in each case.

that the dependence should change again to $\omega^{4}$ at very low $\omega$ due to Rayleigh scattering, as found in LJ glasses ${ }^{10}$ and other glasses like glycerol and some network glasses ${ }^{31}$. In our case the dispersion on the data increases noticeably below $3 \mathrm{THz}$ in longitudinal excitations and may admit an $\omega^{4}$ dependence, but at very low frequencies this behavior is lost. This dispersion is due to the very long simulation times needed to compute the eigenfrequencies corresponding to very low wavenumbers, and does not allows us to confirm or discard the $\omega^{4}$ dependence. In the case of transverse excitations the characteristic frequencies are always lower than for longitudinal excitations, and the required simulation times are even longer. Accordingly, the dispersion of the data that may correspond to an $\omega^{4}$ dependence appears at lower frequencies than for longitudinal excitations.

Figure 6 shows the reduced density of states (DOS) of the simulated glasses. The intensity of the Boson peak is very similar in $\mathrm{Zr}_{50} \mathrm{Cu}_{40} \mathrm{Al}_{10}$ regardless the quenching rate and decreases very slightly in $\mathrm{Pd}_{82} \mathrm{Si}_{18}$ as the quenching rate decreases. A decrease of the excess density of states is expected in more stable glasses and it is closely related to a change in the shear modulus ${ }^{32}$. In this sense, the slight decrease in the DOS of $\mathrm{Pd}_{82} \mathrm{Si}_{18}$ is consequent with the small increase of shear modulus detected in this case.

The two most salient features of the dispersion relation are that there is not any signature of softening in the low- $q$ region, in fact the $c_{L, T}(q \rightarrow 0)$ behavior is strikingly flat, and that the hardening transition appears at different wavelengths for longitudinal and transverse 


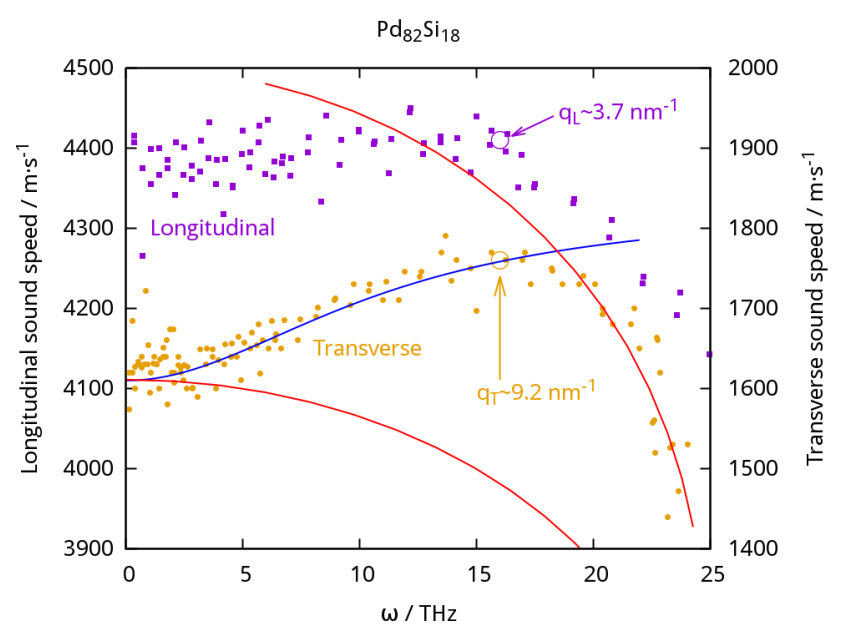

FIG. 7. Phase sound speed of $\mathrm{Pd}_{82} \mathrm{Si}_{18}$ quenched at $10^{12} \mathrm{~K} \cdot \mathrm{s}^{-1}$ vs. frequency. The red solid lines show the behavior of two simple sinusoidal dispersion relations for the transverse sound speed obtained with different values of the shear modulus. The blue line shows the behavior of a viscoelastic-like transition.

polarizations. However, when plotted as a function of frequency - Figure 7, for $\mathrm{Pd}_{82} \mathrm{Si}_{18}$ - the behavior of both phase sound speeds shows a common trend: both increase up to a frequency $\sim 16 \mathrm{THz}$ - hardening effect -, and then drop. This decrease is related to the bending of the dispersion curve when approaching the pseudo-Brillouin zone boundary, at $\approx 15 \mathrm{~nm}^{-1}$. Note that the Debye frequency $\omega_{D} \sim 23 \mathrm{THz}$ in both glasses. Remarkably, the values of the wavenumbers at which the sound speeds show their maxima, indicated in Figure 7, are very different for longitudinal and transverse excitations. These facts suggest that the anomalies in the phase sound speed are due to a frequency effect rather than to any spatial feature.

It order to ensure that these results are actual features of the glass dynamics, not due to the EAM potential used in the simulations, crystalline $\mathrm{ZrCuAl}$ - Fd3̄m space group was also simulated at $300 \mathrm{~K}$ using the same potential. Figure 8 shows the longitudinal and transverse phase sound speeds in this case, plotted up to $5 \mathrm{~nm}^{-1}$; above this wavelenght the acoustic and optic branches merge and it is not possible to determine precisely the sound speed. Both sound speeds are much higher than in the $\mathrm{Zr}_{50} \mathrm{Cu}_{40} \mathrm{Al}_{10}$ glass; in particular, the transverse sound speed is almost doubled. Furthermore, both sound speeds are well fitted to simple sinusoidal relations of dispersion such as 


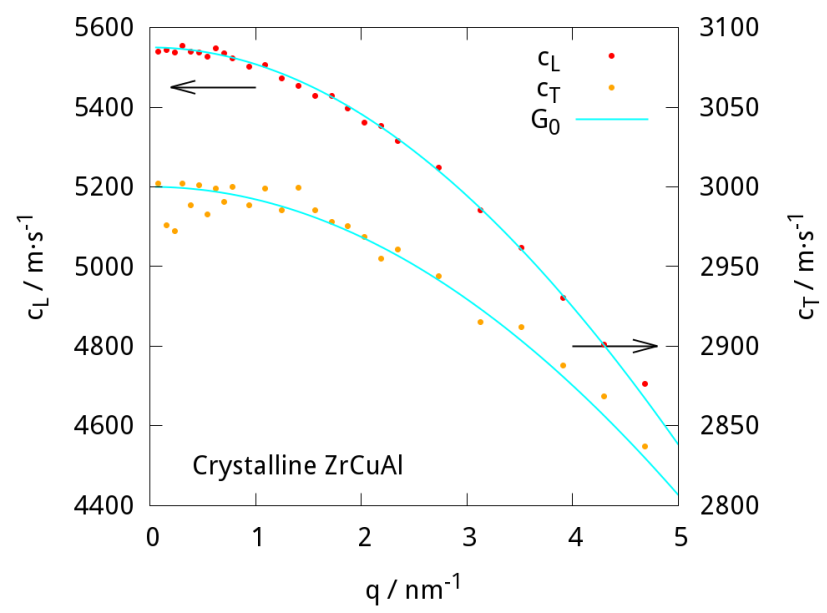

FIG. 8. Phase sound speeds computed in crystalline $\mathrm{ZrCuAl}$ at $300 \mathrm{~K}$. Solid lines show the fit to simple sinusoidal relations of dispersion for the longitudinal and transverse excitations.

$$
\omega_{L, T}(q)=A_{L, T} \sin \left(\frac{q a}{2}\right)
$$

where $L$ and $T$ stand for longitudinal and transverse, $\omega_{L, T}(q)$ are the eigenfrequencies of $L$ and $T$ excitations with wavevector $q$ and $a$ is the lattice parameter. Thus, the crystalline structure shows no signs of hardening, and an explanation for the anomalies observed should be related to the glass structure.

\section{MODEL OF THE DISPERSON RELATION}

Phonon dispersion curves in binary metallic glasses were computed by using pseudopotential methods ${ }^{2933}$, obtaining simple dispersion relations close to the classical sinusoidal form

$$
\omega_{L, T}(q)=A_{L, T} \sin \left(\frac{\pi q}{Q_{L, T}}\right)
$$

where $Q_{L}$ is somewhat less than the position of the first diffraction peak - i.e. the wavevector at the boundary of the pseudo-Brillouin zone - and $Q_{T}>Q_{L}$. The longitudinal and transverse amplitudes are given by

$$
A_{L}=\frac{Q_{L}}{\pi} \sqrt{\frac{K+\frac{4}{3} G}{\rho}}, \quad A_{T}=\frac{Q_{T}}{\pi} \sqrt{\frac{G}{\rho}}
$$


$K$ and $G$ being the bulk and shear modulus, and $\rho$ the density of the material. However, these computations did not predict any hardening of the phase sound speed.

The red lines in Figure 7 show the expected behavior of simple sinusoidal relations of dispersion corresponding to two values of the shear modulus $G$. The lower one is drawn for the value of $\mathrm{G}$ corresponding to the macroscopic value of $c_{T}$; we will call it soft. The discrepancy with the simulated data is striking. On the contrary, the high frequency region is well fitted by a simple sinusoidal relation of dispersion computed for a much higher value of the shear modulus than for $q \rightarrow 0$; will be referred as hard. In order to model the transition from soft to hard dispersion relations we propose a simple Maxwell model of a viscoelastic transition. In the Maxwell model the bulk and shear modulus depend on the frequency,

$$
\begin{aligned}
& K(\omega)=K_{0}+\left(K_{\infty}-K_{0}\right) \frac{\omega^{2} \tau^{2}}{1+\omega^{2} \tau^{2}} \\
& G(\omega)=G_{0}+\left(G_{\infty}-G_{0}\right) \frac{\omega^{2} \tau^{2}}{1+\omega^{2} \tau^{2}}
\end{aligned}
$$

where $K_{0, \infty}$ and $G_{0, \infty}$ are the bulk and shear modulus at macroscopic $(\omega \rightarrow 0)$ and infinite frequencies, respectively. The blue line in 7 is a qualitative fit of such a viscoelastic transition to the low frequency region of the transverse phase sound speed. The parameter $\tau$ corresponds to the characteristic relaxation time of the Maxwell model, and it allows us to define a corresponding threshold frequency $\omega_{h}=\tau^{-1}$.

The resulting relations of dispersion can be solved analytically. The phase speed of longitudinal and transverse oscillations is then

$$
c_{L, T}\left(q_{L, T}\right)=\frac{\omega_{L, T}\left(q_{L, T}\right)}{q_{L, T}}
$$

The dark solid lines in figure 4 show the fit of $c_{L}$ and $c_{T}$ by using equations (5)-(7). The fit was performed simultaneously on both speeds, the fitting parameters being $K_{0, \infty}, G_{0, \infty}$, $Q_{L, T}$ and $\tau$. Table II shows the results of the fits. The fitted value of $Q_{L}$ for all materials and quenching rates is somewhat lower than $Q_{\text {Lexp }}$, the $q$ value of the first peak of the static structure factor $S(q)$ shown in figure 2, as expected. The magnitude of the hardening due to the frequency dependent elastic modulus is noted by comparison with the expected phase speed obtained without the frequency correction (cyan solid line in Figure 4). While the bulk modulus remain constant at all frequencies, the shear modulus increase in the infinite frequency limit by $50 \%$ in $\mathrm{Zr}_{50} \mathrm{Cu}_{40} \mathrm{Al}_{10}$ and is more than doubled in $\mathrm{Pd}_{82} \mathrm{Si}_{18}$. This fact explains why the effect is pronounced in the transverse phase sound speed, while it is much 
more subtle in the longitudinal sound speed. The frequency $\omega_{h}$, associated to the relaxation time $\tau$, lays just above the Boson Peak for both materials, shown in figure 6 .

The frequencies $\omega_{\Gamma,(L, T)}$ of the $\omega^{2} / \omega$ crossover, determined from Figure 5 are also shown in Table II. A remarkable correspondence can be seen between the transverse crossover frequency $\omega_{\Gamma, T}$ and the determined relaxation frequency $\omega_{h}$ in $\mathrm{Zr}_{50} \mathrm{Cu}_{40} \mathrm{Al}_{10}$, pointing to a link between the hardening process and the dynamics of transverse excitations. On the contrary, $\omega_{h}>\omega_{\Gamma, T}$ in $\mathrm{Pd}_{82} \mathrm{Si}_{18}$, but as previously indicated the behavior of high frequency excitations is strongly affected by the presence of Einstein oscilators in this case.

\section{DISCUSSION AND CONCLUSIONS}

The above results confirm that there is a substantial change in the phonon propagation mode in metallic glasses in the Terahertz range, connected to a strong increase on the shear modulus. The frequency of the transition appears at the end of the Boson peak. The QLV model developed by Schober ${ }^{7}$ predicts the increase of the slope in the phonon dispersion relation at a frequency related to the Boson peak. Below this frequency, coupled QLV dominate the phonon spectrum and are responsible of the Boson peak, while above it quasilocalized vibrations become uncoupled. The model predicts also a $\omega^{4}$ dependence of the sound speed attenuation for the low frequency region which was insinuated here, though it could not be completely confirmed. On the whole, our results are consistent with the QLV

TABLE II. Fit parameters and magnitudes determined from MD simulation.

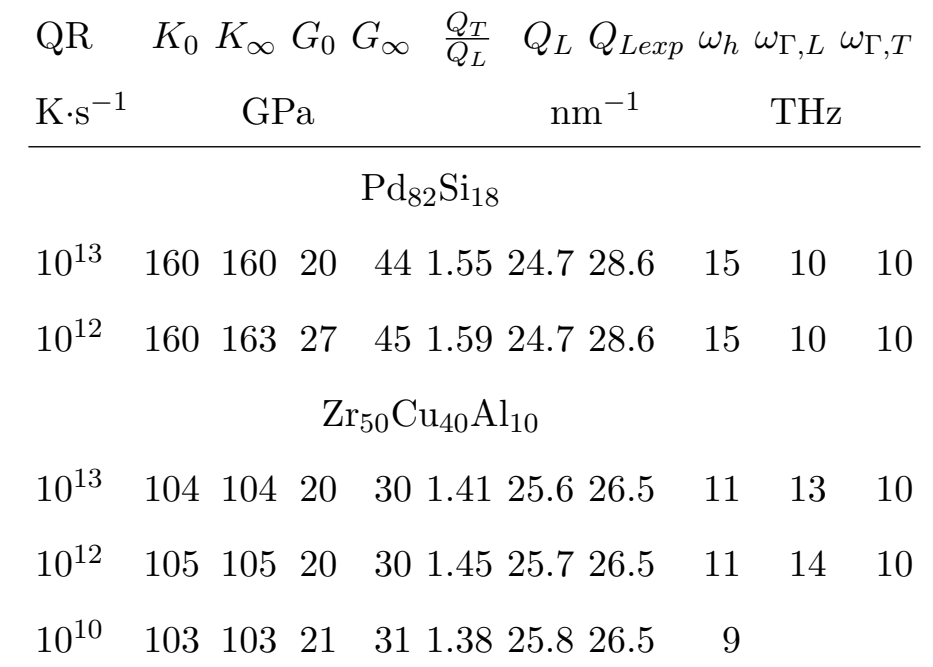


picture, though a further structural characterization is needed to assure the validity of the QLV description.

The ability of a simple Maxwell model to describe this soft/hard transition can be interpreted in terms of the presence of a relaxation process; vibrations faster than the characteristic relaxation see a stiffer structure. The presence of elastic relaxations or non-affine rearrangements, with characteristic times of the order of the picosecond, is a known characteristic of amorphous structures ${ }^{34}$. They have been estimated to be responsible of a reduction of around 10\% (Bulk) and 30\% (Shear) of the elastic constants respect to the "Born terms" calculated considering only affine deformations ${ }^{3520}$. In this picture, our results would indicate that the interaction of the non-affine rearrangements with the propagating vibration modes would be not only responsible of the change in elastic constants but also responsible of the $\omega^{2}$ damping behavior. This coincides with the view suggested by Baldi et al. ${ }^{8}$ of a transition between glass-like modes, at low wavenumbers, and crystal-like modes, at high wavenumbers. The different frequency dependencies of the sound attenuation coefficients are also the signatures of the different phonon modes, in a similar way than what was found in amorphous silica and polycrystalline quartz. If the transition between the "hard" and "soft" elastic regimes at the $\mathrm{THz}$ frequencies indicates an onset of glassy dynamics, this may be related to the onset of the fast secondary relaxation processes originating the high-frequency loss detected experimentally in glass susceptibility ${ }^{36,37}$.

Not less important, our results in the macroscopic limit - $q \rightarrow 0$ - solve the controversy about the behavior of the sound speed in glasses. Both the longitudinal and transverse sound speeds have constant values up to the nm lengthscale. In fact, the glass behaves as a continuum both in the macroscopic region and in the Boson Peak region. It is above the Boson Peak frequency when the changes in the phonon propagation mode become determinant.

Several questions remain still open. The first is why simulations using EAM offer results different than those performed using LJ and SS potentials. As the shear modulus is responsible for the frequency of transverse excitations, the specific description of the shear forces plays a dominant role in their behavior. Being a two-body potential, LJ systems have shear interactions very different from realistic metallic systems, and this may explain the observed differences. The second question is that anomalies of the phonon spectra of metallic glasses have been generally associated to the existence of compositional and structural inhomogeneities. However, a single relaxation frequency $\omega_{h}$ governs both longitudinal 
and transverse relations of dispersion, close to the frequency $\omega_{\Gamma,(L, T)}$ at which the phonon damping behavior also changes. This behavior, summed to the fact that no signs of any well defined lengthscale were found in our simulations, prevents us to relate the transition between different phonon modes to spatial inhomogeneities. Finally, it would be very interesting to identify the quasi-localized vibrations in the molecular dynamics simulations, or correlate them with some structural features in order to confirm the validity of Schober's model.

In summary, numerical simulation of the phonon spectra of metallic glasses confirms a change in the phonon regime in the Terahertz range. Below this frequency range, both in the macroscopic range and in the Boson Peak region, the glass behaves as a continuum and the sound speed is constant. In the Terahertz range the phonon dynamics is governed by a substantial increase of the shear modulus. The experimentally reported hardening of the sound speed in the nanometer range may be due to the uncoupling of quasi localized vibrations marking the end of the Boson peak. This "viscoelastic-like" transition is well described by a single relaxation frequency. The dispersion of the IXS results obtained in metallic glasses may be consequence of different relaxation frequencies in the different amorphous structures.

\section{ACKNOWLEDGMENTS}

Work funded by MINECO (FIS2014-54734-P), and Generalitat de Catalunya (2014SGR00581). Simulations were performed in Barcelona Supercomputer Center, grants FI-2013-1-0019 and QCM-2015-2-0017.

* Daniel.Crespo@upc.edu; http://mie.esab.upc.es/mmn

1 H. W. Sheng, W. K. Luo, F. M. Alamgir, J. M. Bai, and E. Ma, Nature 439, 419 (2006).

2 Y. Zhang, C. Z. Wang, F. Zhang, M. I. Mendelev, M. J. Kramer, and H. K. M., Applied Physics Letters 105, 151910 (2014).

3 T. Ichitsubo, S. Hosokawa, K. Matsuda, E. Matsubara, N. Nishiyama, S. Tsutsui, and A. Q. R. Baron, Phys Rev B 76, 140201 (2007). 
4 T. Ichitsubo, W. Itaka, E. Matsubara, H. Kato, S. Biwa, S. Hosokawa, K. Matsuda, J. Saida, O. Haruyama, Y. Yokoyama, H. Uchiyama, and A. Q. R. Baron, Phys Rev B 81, 172201 (2010).

5 P. Bruna, J. Serrano, E. Pineda, M. Duarte, K. Zhao, W. Wang, and D. Crespo, Intermetallics 30, 148 (2012).

6 P. Bruna, G. Baldi, E. Pineda, J. Serrano, J. B. Suck, D. Crespo, and G. Monaco, J. Chem Phys 135, 101101 (2011).

7 H. Schober, Journal of Non-Crystalline Solids 357, 501 (2011).

8 G. Baldi, M. Zanatta, E. Gilioli, V. Milman, K. Refson, B. Wehinger, B. Winkler, A. Fontana, and G. Monaco, Physical Review Letters 110, 185503 (2013).

9 P. Lunkenheimer, L. C. Pardo, M. Köhler, and A. Loidl, Phys. Rev. E 77, 031506 (2008).

10 G. Monaco and S. Mossa, Proc. Natl. Acad. Sci. USA 106, 16907 (2009).

11 M. Li, C. Z. Wang, S. G. Hao, M. J. Kramer, and K. M. Ho, Phys. Rev. B 80, 184201 (2009).

12 A. Marruzzo, W. Schirmacher, A. Fratalocchi, and G. Ruocco, Sci. Rep 3 (2013).

13 G. Monaco and V. M. Giordano, Proceedings of the National Academy of Sciences of the United States of America 106, 3659 (2009).

14 H. Mizuno, S. Mossa, and J. Barrat, EPL (Europhysics Lett.) 104, 56001 (2014).

15 H. Mizuno, S. Mossa, and J. Barrat, Proc. Natl. Acad. Sci. U.S.A. 111, 11949 (2014).

16 G. Ruocco, F. Sette, R. Di Leonardo, G. Monaco, M. Sampoli, T. Scopigno, and G. Viliani, Phys. Rev. Lett. 84, 5788 (2000).

17 T. Voigtmann, Physical Review Letters 101, 095701 (2008).

18 L. Berthier and G. Tarjus, Physical Review Letters 103, 170601 (2009).

19 P. Derlet, R. Maaß, and J. Löffler, The European Physical Journal B 85, 148 (2012).

20 F. Leonforte, R. Boissière, A. Tanguy, J. P. Wittmer, and J. L. Barrat, Physical Review B 72, $224206(2005)$.

21 Y. H. Liu, D. Wang, K. Nakajima, W. Zhang, A. Hirata, T. Nishi, A. Inoue, and M. W. Chen, Physical Review Letters 106, 125504 (2011).

22 M. S. Daw and M. I. Baskes, Phys Rev B 29, 6443 (1984).

23 Y. Q. Cheng, E. Ma, and H. W. Sheng, Physical Review Letters 102, 245501 (2009).

24 H. Sheng, "Pdsi eam potentials, https://sites.google.com/site/eampotentials/home/pdsi," (2011).

25 S. Plimpton, J. Comp. Phys. 117, 1 (1995). 
26 T. Scopigno, J. B. Suck, R. Angelini, F. Albergamo, and G. Ruocco, Phys Rev Lett. 96, 135501 (2006).

27 Z. Zhou, C. Uher, D. Xu, W. L. Johnson, W. Gannon, and M. C. Aronson, Applied Physics Letters 89, 031924 (2006).

28 S. Hosokawa et al. (ISMANAM, Paris, 2015).

29 J. Hafner, Physical Review B 27, 678 (1983).

30 J. Hafner and M. Krajci, J. Phys.: Condens. Matter 6, 4631 (1994).

31 A. Matic, D. Engberg, C. Masciovecchio, and L. Börjesson, Physical Review Letters 86, 3803 (2001).

32 H. Shintani and H. Tanaka, Nature Materials 7, 870 (2008).

33 M. V. Vora, Glass Physics and Chemistry 34, 671 (2008).

34 G. Knuyt, L. Deschepper, and L. Stals, Journal of Physics F-Metal Physics 16, 1989 (1986).

35 G. Knuyt and L. Stals, Philosophical Magazine B 64, 299 (1991).

36 K. L. Ngai, S. Capaccioli, D. Prevosto, and L.-M. Wang, The Journal of Physical Chemistry B 119, 12519 (2015).

37 Z. Wang, K. L. Ngai, W. H. Wang, and S. Capaccioli, Journal of Applied Physics 119, 024902 (2016). 